\title{
Modeling Portfolio Optimization Problem by Probability-Credibility Equilibrium Risk Criterion
}

\author{
Ye Wang, Yanju Chen, and YanKui Liu \\ Key Laboratory in Machine Learning \& Computational Intelligence, College of Mathematics \& Information Science, \\ Hebei University, Baoding, Hebei 071002, China
}

Correspondence should be addressed to Yanju Chen; chen-yanju@163.com

Received 3 December 2015; Accepted 15 February 2016

Academic Editor: Kishin Sadarangani

Copyright (C) 2016 Ye Wang et al. This is an open access article distributed under the Creative Commons Attribution License, which permits unrestricted use, distribution, and reproduction in any medium, provided the original work is properly cited.

This paper studies the portfolio selection problem in hybrid uncertain decision systems. Firstly the return rates are characterized by random fuzzy variables. The objective is to maximize the total expected return rate. For a random fuzzy variable, this paper defines a new equilibrium risk value (ERV) with credibility level beta and probability level alpha. As a result, our portfolio problem is built as a new random fuzzy expected value (EV) model subject to ERV constraint, which is referred to as EV-ERV model. Under mild assumptions, the proposed EV-ERV model is a convex programming problem. Furthermore, when the possibility distributions are triangular, trapezoidal, and normal, the EV-ERV model can be transformed into its equivalent deterministic convex programming models, which can be solved by general purpose optimization software. To demonstrate the effectiveness of the proposed equilibrium optimization method, some numerical experiments are conducted. The computational results and comparison study demonstrate that the developed equilibrium optimization method is effective to model portfolio selection optimization problem with twofold uncertain return rates.

\section{Introduction}

Based on mean-variance criterion, Markowitz [1] first established portfolio theory. In Markowitz's mean-variance model, the returns of individual securities are taken as random variables and the expected value and variance of the random return are taken as the investment return and risk, respectively. The mean-variance theory has been accepted widely as a tool for building portfolio optimization problems, and many interesting works have further developed the mean-variance method. Considering that the situation of the expected return is lower than the average value, Markowitz [2] replaced variance with semivariance as the risk measure to describe the risk. Semivariance is an improvement of variance, which allows us to obtain the higher expected return rate under the same level of risk. Konno and Yamazaki [3] used mean absolute deviation model in Tokyo stock market. Simaan [4] showed that estimation error is large in both mean-variance model and mean absolute deviation model and severe in small samples and for investors with high risk tolerance. In order to describe the normal fluctuations in the market, the concept of value-at-risk (VaR) was taken as the risk measure. $\mathrm{VaR}$ is the maximum possible loss of a financial asset or portfolio of securities. In recent years, VaR gradually progresses as a positive risk management tool and plays an increasingly important role in the portfolio optimization problems. Jorion [5] studied $\mathrm{VaR}$ as a risk measure and applied mean-VaR model in finance industry. Mean-variance-skewness models [6,7] were also proposed to study portfolio selection problem. In addition, there are other measures used to characterize the risk [8]. When the restriction that an asset cannot be traded for intervals of uncertain duration results in risk, a model of optimal allocation to liquid and illiquid assets was presented by Ang et al. [9]. Shen et al. [10] discussed a mean-variance portfolio selection problem under a constant elasticity of variance model and expressed explicit expressions of the optimal portfolio strategy, the value function, and the efficient frontier of the mean-variance problem.

The conventional portfolio methods assume that the security returns are random variables. Probability distributions of random variables are usually derived from historical 
data. However, in the real investment environment, the security returns often present vagueness and ambiguity. Since the seminal works of Zadeh [11, 12], many achievements have been made based on fuzzy theory. Watada [13] discussed portfolio selection by using fuzzy decision theory. Tanaka and Guo [14] used possibility distributions to model the uncertain returns. Inuiguchi and Ramík [15] exemplified the advantages and disadvantages of fuzzy mathematical programming approaches in the setting of an optimal portfolio selection problem. When the returns of assets are taken as trapezoidal fuzzy numbers, a possibilistic approach for selecting portfolios with the highest utility value was introduced by Carlsson et al. [16]. Considering transaction costs, Fang et al. [17] proposed a portfolio rebalancing model based on fuzzy decision theory. Mean-variance models were constructed for fuzzy portfolio selection problems by Chen et al. [18] and Zhang et al. [19]. Qin et al. [20] discussed the Kapur cross-entropy minimization model for portfolio selection problem under fuzzy environment. Dastkhan et al. [21] studied a linguistic-based portfolio selection model by weighted max-min operator. $\mathrm{Wu}$ and Liu [22] developed a robust method to describe fuzzy returns by employing parametric possibility distributions. Chen et al. [23] applied the absolute semideviation as a new risk measure and developed three classes of fuzzy portfolio optimization models. Kamdem et al. [24] introduced notions of moments and semimoments for credibilistic portfolio problems to measure the leptokurtocity of credibilistic portfolio return and presented a mean-variance-skewnesssemikurtosis model. Mehlawat and Gupta [25] addressed fuzzy portfolio selection problem from a perspective of chance constrained multiobjective programming. Deng and $\mathrm{Li}$ [26] proposed a biobjective nonlinear portfolio selection model, which aimed to maximize the future expected return and minimize the future expected risk. They also proposed a gradually tolerant constraint method to solve this model. Li et al. [27] developed a fuzzy portfolio selection model with background risk. Vercher and Bermúdez [28] introduced a cardinality constrained multiobjective optimization problem for generating efficient portfolios within a fuzzy mean absolute deviation framework. Considering transaction cost, Chen and Wang [29] proposed a two-stage fuzzy model for portfolio selection problem. For fuzzy portfolio selection problems, there are many other different optimization methods.

In modern financial markets, there is not only randomness but also fuzziness that affects the total decision-making process. Based on the above works, this paper models the portfolio selection problem where randomness and fuzziness are considered simultaneously. Huang [30] studied portfolio selection in a random fuzzy environment and proposed two portfolio selection models with random fuzzy returns. We adopt the equilibrium optimization method [31] to model portfolio selection problems. In order to integrate uncertainties resulting from both subjective consciousness and objective factors, random fuzzy variables [32] are used to characterize the return rates. In this situation, the security return rates are assumed to be random variables with fuzzy information. This paper employs the expected value (EV) [33] to represent the investment return and introduces a new index called ERV for random fuzzy return rate to measure the investment risk. Then a random fuzzy EV-ERV model is proposed for portfolio selection problem. Since optimization models with twofold uncertainty are difficult to solve, the heuristic algorithms are often designed to solve the proposed optimization models. An advantage of the proposed equilibrium optimization framework is to turn the original equilibrium portfolio optimization problem to a computationally tractable problem. Firstly, the proposed model is translated into an equivalent credibilistic optimization model. Then the credibilistic model is reduced to its equivalent deterministic convex programming models under mild assumptions on fuzzy parameters.

Compared with the existing literature, the main contributions of this paper consist of the following three aspects. Firstly, this paper defines a new ERV with credibility level beta and probability level alpha to measure the investment risk. The adopted risk measure quantifies the uncertainties of randomness and fuzziness simultaneously. This method shows the qualitative and quantitative analysis about the uncertainty of return rate. The proposed EV-ERV model is a useful optimization method for a practical investor. Secondly, the global optimal solution of the proposed EV-ERV model is obtained. In the case that the randomness of uncertain return rates follows normal distributions with deterministic covariance matrix, and the fuzziness is characterized by trapezoidal fuzzy variables, triangular fuzzy variables, or normal fuzzy variables, the proposed EV-ERV model is transformed into its deterministic convex programming models, which can be solved by general purpose optimization software. Thirdly, when the randomness of uncertain return rate vector follows multivariate normal distribution, the covariance matrix can reflect the interactions and correlation degrees among securities.

This paper is organized as follows. In Section 2, after introducing a new index called ERV for random fuzzy variable, the random fuzzy EV-ERV model is proposed for portfolio selection problems. In Section 3, under mild assumptions on possibility and probability distributions, the EV-ERV equilibrium optimization model is proved to be a convex programming. Section 4 deals with the equivalent deterministic convex programming models under three situations. In Section 5, we perform some comparison study via numerical experiments, give the sensitivity analysis about model parameters, and show the relationship between ERV and EV. The last section gives the conclusions of the paper.

\section{Equilibrium Portfolio Selection Problem}

2.1. Random Fuzzy Variable. Let $\Gamma$ be an abstract space of generic elements $\gamma$ and $\mathscr{P}(\Gamma)$ the power set of $\Gamma$. If Pos is a possibility measure on $\mathscr{P}(\Gamma)$, then the credibility measure $\mathrm{Cr}$ is defined as follows [34]:

$$
\operatorname{Cr}(A)=\frac{1}{2}\left(1+\operatorname{Pos}(A)-\operatorname{Pos}\left(A^{c}\right)\right), \quad A \in \mathscr{P}(\Gamma),
$$

where $A^{c}=\Gamma \backslash A$. The triplet $(\Gamma, \mathscr{P}(\Gamma), \mathrm{Cr})$ is called a credibility space. 
Definition 1 (see [32]). Let $(\Gamma, \mathscr{P}(\Gamma), \mathrm{Cr})$ be a credibility space and $(\Omega, \Sigma, \operatorname{Pr})$ a probability space. If $\xi$ is a map defined on $\Gamma \times \Omega$ such that, for each fixed $\gamma \in \Gamma, \xi(\gamma, \omega)$ as a function of $\omega$ is a random variable defined on the probability space $(\Omega, \Sigma, \operatorname{Pr})$, then one calls $\xi$ a random fuzzy variable.

If $\xi_{1}, \xi_{2}, \ldots, \xi_{n}$ are random fuzzy variables defined on the space $\Gamma$, then $\xi=\left(\xi_{1}, \xi_{2}, \ldots, \xi_{n}\right)$ is a random fuzzy vector defined on the space $\Gamma$.

A multivariate normal distribution $\mathcal{N}(\mu, \Sigma)$ with uncertain mean $\mu$ and covariance $\Sigma$ is an example of random fuzzy vector. In this case, the components of $\mu$ and $\Sigma$ are represented as fuzzy variables with known possibility distributions.

Definition 2 (see [33]). Let $\xi$ be a random fuzzy variable. The expected value $E[\xi]$ of $\xi$ is defined as the following fuzzy integral:

$$
\begin{aligned}
E[\xi]= & \int_{0}^{+\infty} \operatorname{Cr}\left\{\gamma \in \Gamma \mid E\left[\xi_{\gamma}\right] \geq r\right\} d r \\
& -\int_{-\infty}^{0} \operatorname{Cr}\left\{\gamma \in \Gamma \mid E\left[\xi_{\gamma}\right] \leq r\right\} d r,
\end{aligned}
$$

where $E\left[\xi_{\gamma}\right]$ is the expected value of random variable $\xi_{\gamma}$ for any given $\gamma \in \Gamma$.

Definition 3 (see [31]). Let $\xi=\left(\xi_{1}, \xi_{2}, \ldots, \xi_{n}\right)$ be a random fuzzy vector, and let $f_{j}: \mathfrak{R}^{\mathfrak{n}} \rightarrow \mathfrak{R}$ be continuous function for $j=1,2, \ldots, m$. Then the equilibrium chance $\mathrm{Ch}$ of a random fuzzy event is defined as

$$
\begin{aligned}
\operatorname{Ch} & \left\{f_{j}(\xi) \leq 0, j=1,2, \ldots, m\right\}=\sup _{(\alpha, \beta) \in[0,1]^{2}}\{\alpha \wedge \beta \mid \\
& \operatorname{Cr}\left\{\gamma \in \Gamma \mid \operatorname{Pr}\left\{f_{j}(\xi(\gamma)) \leq 0, j=1,2, \ldots, m\right\} \geq \alpha\right\} \\
& \geq \beta\} .
\end{aligned}
$$
form:

Equation (3) can be rewritten as the following equivalent

$$
\begin{gathered}
\operatorname{Ch}\left\{f_{j}(\xi) \leq 0, j=1,2, \ldots, m\right\}=\sup _{\alpha \in[0,1]}\{\alpha \wedge \operatorname{Cr}\{\gamma \\
\left.\left.\quad \in \Gamma \mid \operatorname{Pr}\left\{f_{j}(\xi(\gamma)) \leq 0, j=1,2, \ldots, m\right\} \geq \alpha\right\}\right\} .
\end{gathered}
$$

According to the property of equilibrium chance, in the case of $\alpha=\beta$, one has

$$
\begin{gathered}
\operatorname{Cr}\left\{\gamma \mid \operatorname{Pr}\left\{\xi_{\gamma} \geq z\right\} \geq \alpha\right\} \geq \alpha \Longleftrightarrow \\
\operatorname{Ch}\{\xi \geq z\} \geq \alpha .
\end{gathered}
$$

We next introduce the equilibrium risk value for a random fuzzy variable.

Definition 4 . Let $\xi$ be a random fuzzy variable. The equilibrium risk value of $\xi$ is defined as

$$
\begin{aligned}
& \operatorname{VaR}_{(\alpha, \beta)}(\xi) \\
& \quad=\sup \left\{z \mid \operatorname{Cr}\left\{\gamma \mid \operatorname{Pr}\left\{\omega \mid \xi_{\gamma}(\omega) \geq z\right\} \geq \alpha\right\} \geq \beta\right\},
\end{aligned}
$$

where $\alpha, \beta$ are prescribed confidence levels and take their values in the unit interval $[0,1]$.

In (6), the meanings of parameters $\alpha$ and $\beta$ are different: the parameter $\alpha$ represents probability level, while the parameter $\beta$ represents credibility level.

If the random fuzzy variable $\xi$ reduces to a random variable, then the equilibrium risk value defined by (6) reduces to

$$
\xi_{\text {sup }}(\alpha)=\sup \{z \mid \operatorname{Pr}\{\omega \mid \xi(\omega) \geq z\} \geq \alpha\}
$$

which is the $\alpha$-optimistic value of random variable $\xi$. On the other hand, if the random fuzzy variable $\xi$ reduces to a fuzzy variable, then the equilibrium risk value defined by (6) reduces to

$$
\xi_{\text {sup }}(\beta)=\sup \{z \mid \operatorname{Cr}\{\gamma \mid \xi(\gamma) \geq z\} \geq \beta\}
$$

which is the $\beta$-optimistic value of fuzzy variable $\xi$. Therefore, the equilibrium risk value of random fuzzy variable is a natural extension of both the optimistic value of random variable and the optimistic value of fuzzy variable.

2.2. Formulation of Equilibrium Optimization Model. With the rapid development of the economic and society, more and more investors realize the importance of portfolio optimization problem under uncertainty. They hope to use the limited funds to get the maximum benefit and, at the same time, to minimize the investment risk as much as possible. However, under the market economy, there are various uncertain factors to affect investment market. Thus, the uncertainties should be taken into account during the modeling process.

A rational investor should pursuit the maximum profit with the minimum risk. However, in real investment process, increasing the return, the investors have to tolerate greater risk; the lower risk corresponds to the less return. That is, investors need to make a trade-off between return and risk. Assume that the investor has a collection of $n$ optional risky assets. The return rate of investment refers to the result of the net income divided by the initial funds. We use random fuzzy variable $\eta_{i}$ to denote the return rate of risky asset $i$, $i=1,2, \ldots, n$. Then $\eta=\left(\eta_{1}, \eta_{2}, \ldots, \eta_{n}\right)$ is the return rate vector. When the return rates of assets are characterized by joint normal distribution $\mathcal{N}(\mu, \Sigma)$, the fuzzy parameters $\mu$ and $\Sigma$ stand for the average return rate vector and the correlations among the return rates of assets, respectively. Since the parameters $\mu$ and $\Sigma$ often cannot be determined exactly due to the lack of the historical data, it is appropriate to represent the components of $\mu$ and $\Sigma$ as fuzzy variables with known possibility distributions.

Let $x_{i}$ be the investment ratio for risky asset $i$ and let $x=\left(x_{1}, x_{2}, \ldots, x_{n}\right)$ be the investment ratio vector such that $\sum_{i=1}^{n} x_{i}=1$, where $x_{i} \geq 0, i=1,2, \ldots, n$. The vector $x=$ $\left(x_{1}, x_{2}, \ldots, x_{n}\right)$ is called a portfolio. So $\eta^{T} x$ is the return rate during the holding period. We take $E\left[\eta^{T} x\right]$ as objective 
function. Then, based on Definition 2, the objective function is computed by

$$
\begin{aligned}
E\left[\eta^{T} x\right]= & \int_{0}^{+\infty} \operatorname{Cr}\left\{\gamma \in \Gamma \mid E\left[\eta_{\gamma}^{T} x\right] \geq r\right\} d r \\
& -\int_{-\infty}^{0} \operatorname{Cr}\left\{\gamma \in \Gamma \mid E\left[\eta_{\gamma}^{T} x\right] \leq r\right\} d r .
\end{aligned}
$$

We take ERV as the risk index to measure the investment risk. Based on Definition 4, the risk of portfolio $x$ is

$$
\begin{aligned}
& \operatorname{VaR}_{(\alpha, \beta)}\left(\eta^{T} x\right) \\
& \quad=\sup \left\{z \mid \operatorname{Cr}\left\{\gamma \mid \operatorname{Pr}\left\{\omega \mid \eta_{\gamma}^{T}(\omega) x \geq z\right\} \geq \alpha\right\} \geq \beta\right\},
\end{aligned}
$$

where $\alpha, \beta \in[0,1]$ are prescribed confidence levels.

Based on the notations above, if the investor wants to maximize the expected return rate, the equilibrium portfolio optimization problem in hybrid uncertain decision systems is built as the following EV-ERV model:

$$
\begin{array}{ll}
\max & E\left[\eta^{T} x\right] \\
\text { s.t. } & \operatorname{VaR}_{(\alpha, \beta)}\left(\eta^{T} x\right) \geq \kappa \\
& \sum_{i=1}^{n} x_{i}=1 \\
& x_{i} \geq 0, i=1,2, \ldots, n,
\end{array}
$$

where parameter $\kappa$ is the prescribed level of ERV.

Introducing an additional variable $z$, the original EV-ERV model (11) can be equivalently represented as

$$
\begin{array}{ll}
\max & E\left[\eta^{T} x\right] \\
\text { s.t. } & \operatorname{Cr}\left\{\gamma \mid \operatorname{Pr}\left\{\eta_{\gamma}^{T} x \geq z\right\} \geq \alpha\right\} \geq \beta \\
& z \geq \kappa \\
& \sum_{i=1}^{n} x_{i}=1 \\
& x_{i} \geq 0, \quad i=1,2, \ldots, n .
\end{array}
$$

\section{Analysis of the Equilibrium Portfolio Model}

In this section, we discuss the properties of the objective function and constrains in equilibrium portfolio optimization model (12), which facilities finding the appropriate algorithm to solve the model.

3.1. Computing Expected Return Rate. Since the return rates are denoted by the random fuzzy vector $\eta$, for any given $\gamma \in \Gamma, \eta_{\gamma}$ is the random vector $\left(\eta_{1, \gamma}, \eta_{2, \gamma}, \ldots, \eta_{n, \gamma}\right)$. Thus, the expected return rate is calculated by the following formula:

$$
E\left[\eta^{T} x\right]=E_{\gamma}\left[E_{\omega}\left[\eta_{\gamma}^{T} x\right]\right]=E_{\gamma}\left[\sum_{i=1}^{n} x_{i} E_{\omega}\left[\eta_{i, \gamma}\right]\right] .
$$

The first equality in (13) holds true due to the definition of expected value operator of random fuzzy variable [33], and the second equality in (13) is based on the linearity of expected value operator of random variable. Note that $\sum_{i=1}^{n} x_{i} E\left[\eta_{i, \gamma}\right]$ is the linear combination of fuzzy variables $E\left[\eta_{i, \gamma}\right], i=1,2, \ldots, n$. To further compute $E_{\gamma}\left[\sum_{i=1}^{n} x_{i} E_{\omega}\left[\eta_{i, \gamma}\right]\right]$, the independence condition or comonotonicity condition for fuzzy variables $E_{\omega}\left[\eta_{i, \gamma}\right], i=1,2, \ldots, n$ is required. We next discuss the two situations, respectively.

Case 1 (independence condition [35]). According to [35], the expected value operator of fuzzy variable has the property of independence linearity. Based on this property, if $E\left[\eta_{1, \gamma}\right], E\left[\eta_{2, \gamma}\right], \ldots, E\left[\eta_{n, \gamma}\right]$ are mutual independent, one has

$$
\begin{aligned}
E_{\gamma}\left[\sum_{i=1}^{n} x_{i} E_{\omega}\left[\eta_{i, \gamma}\right]\right] & =\sum_{i=1}^{n} x_{i} E_{\gamma}\left[E_{\omega}\left[\eta_{i, \gamma}\right]\right] \\
& =\sum_{i=1}^{n} x_{i} E\left[\xi_{i}\right] .
\end{aligned}
$$

Case 2 (comonotonicity condition [34]). According to [34, Theorem 1], the expected value operator of fuzzy variable has the property of comonotonic linearity. Based on this property, if $E\left[\eta_{1, \gamma}\right], E\left[\eta_{2, \gamma}\right], \ldots, E\left[\eta_{n, \gamma}\right]$ are comonotonic, one has

$$
\begin{aligned}
E_{\gamma}\left[\sum_{i=1}^{n} x_{i} E_{\omega}\left[\eta_{i, \gamma}\right]\right] & =\sum_{i=1}^{n} x_{i} E_{\gamma}\left[E_{\omega}\left[\eta_{i, \gamma}\right]\right] \\
& =\sum_{i=1}^{n} x_{i} E\left[\xi_{i}\right] .
\end{aligned}
$$

In the above two cases, the expected value operator of fuzzy variable has linear property, $E\left[\eta^{T} x\right]=\sum_{i=1}^{n} x_{i} E\left[\eta_{i}\right]$. In general case, we may compute $E_{\gamma}\left[\sum_{i=1}^{n} x_{i} E_{\omega}\left[\eta_{i, \gamma}\right]\right.$ by using fuzzy simulation or approximation method $[33,36]$ based on the possibility distributions of fuzzy variables $E_{\omega}\left[\eta_{i, \gamma}\right], i=$ $1,2, \ldots, n$.

3.2. Processing Equilibrium Risk Value. In this subsection, we will handle the following probability constraint in model (12):

$$
\operatorname{Pr}\left\{\eta_{\gamma}^{T} x \geq z\right\} \geq \alpha,
$$

where the fuzzy parameter $\gamma \in \Gamma$ is given in advance.

It is assumed that random vector $\eta_{\gamma}$ follows a multivariate normal distribution $\mathcal{N}\left(\mu_{\gamma}, \Sigma_{\gamma}\right)$. The covariance matrix $\Sigma_{\gamma}$ is a symmetric positive semidefinite matrix. So there exists a lower triangular and nonsingular matrix $D_{\gamma}, \mu_{\gamma} \in R^{n}$, and a random vector $\xi$ with components being independent standard normal random variables such that $\eta_{\gamma}=D_{\gamma} \xi+\mu_{\gamma}$. Thus, one has $E\left[\eta_{\gamma}\right]=\mu_{\gamma}$ and $\Sigma_{\gamma}=D_{\gamma} D_{\gamma}^{T}$. If $\Sigma_{\gamma}$ is positive definite, then the multivariate normal distribution is nondegenerate. This case appears if and only if $D_{\gamma}$ has full row rank. Otherwise the distribution is degenerate or singular. The multivariate normal distribution is uniquely determined by the expected value vector $\mu_{\gamma}$ and the covariance matrix $\Sigma_{\gamma}$. 
If the multivariate normal distribution is nondegenerate, then it is absolutely continuous. Thus, one has $\eta_{\gamma}^{T} x \sim \mathcal{N}\left(\mu_{\gamma}^{T} x\right.$, $\left.x^{T} D_{\gamma} D_{\gamma}^{T} x\right)$.

Let $G(x)=\operatorname{Pr}\left\{\eta_{\gamma}^{T} x \geq z\right\}$. If $\sqrt{x^{T} D_{\gamma} D_{\gamma}^{T} x}>0$, by standardization, $G(x)$ has the following equivalent representation:

$$
\begin{aligned}
\operatorname{Pr}\left\{\eta_{\gamma}^{T} x \geq z\right\} & =\operatorname{Pr}\left\{\frac{\eta_{\gamma}^{T} x-\mu_{\gamma}^{T} x}{\sqrt{x^{T} D_{\gamma} D_{\gamma}^{T} x}} \geq \frac{z-\mu_{\gamma}^{T} x}{\sqrt{x^{T} D_{\gamma} D_{\gamma}^{T} x}}\right\} \\
& =\Phi\left(\frac{\mu_{\gamma}^{T} x-z}{\sqrt{x^{T} D_{\gamma} D_{\gamma}^{T} x}}\right) .
\end{aligned}
$$

Taking all the cases of $G(x)$ into account, the following analytical expression is obtained:

$$
\begin{aligned}
& G(x) \\
& = \begin{cases}1, & \text { if } x^{T} D_{\gamma} D_{\gamma}^{T} x=0, \mu_{\gamma}^{T} x \geq z, \\
0, & \text { if } x^{T} D_{\gamma} D_{\gamma}^{T} x=0, \mu_{\gamma}^{T} x<z, \\
\Phi\left(\frac{\mu_{\gamma}^{T} x-z}{\sqrt{x^{T} D_{\gamma} D_{\gamma}^{T} x}}\right), & \text { if } x^{T} D_{\gamma} D_{\gamma}^{T} x \neq 0 .\end{cases}
\end{aligned}
$$

For any given $\gamma \in \Gamma$, if $x^{T} D_{\gamma} D_{\gamma}^{T} x \neq 0$, then one has

$$
\begin{gathered}
G(x) \geq \alpha \Longleftrightarrow \\
\Phi\left(\frac{\mu_{\gamma}^{T} x-z}{\sqrt{x^{T} D_{\gamma} D_{\gamma}^{T} x}}\right) \geq \alpha \Longleftrightarrow \\
\frac{\mu_{\gamma}^{T} x-z}{\sqrt{x^{T} D_{\gamma} D_{\gamma}^{T} x}} \geq \Phi^{-1}(\alpha) \Longleftrightarrow \\
\mu_{\gamma}^{T} x \geq \Phi^{-1}(\alpha) \sqrt{x^{T} D_{\gamma} D_{\gamma}^{T} x}+z .
\end{gathered}
$$

From the above analysis, if $E\left[\eta_{1, \gamma}\right], E\left[\eta_{2, \gamma}\right], \ldots, E\left[\eta_{n, \gamma}\right]$ are comonotonic or mutual independent, then model (12) can be turned into the following credibilistic programming problem:

$$
\begin{array}{ll}
\max & \sum_{i=1}^{n} x_{i} E\left[\mu_{i}\right] \\
\text { s.t. } & \operatorname{Cr}\left\{\gamma \mid \mu_{\gamma}^{T} x \geq \Phi^{-1}(\alpha) \sqrt{x^{T} D_{\gamma} D_{\gamma}^{T} x}+z\right\} \geq \beta \\
& z \geq \kappa \\
& \sum_{i=1}^{n} x_{i}=1 \\
& x_{i} \geq 0, i=1,2, \ldots, n .
\end{array}
$$

3.3. The Convexity of Feasible Region. Section 3.2 has dealt with the probability risk. In this subsection, we will address the equivalent representation of credibilistic constraint and discuss the convexity of feasible region.

Let $\mathscr{C}=\left\{x \mid \operatorname{Cr}\left\{\mu^{T} x \geq \Phi^{-1}(\alpha) \sqrt{x^{T} D D^{T} x}+z\right\} \geq \beta\right\}$. In real-life portfolio selection problems, small confidence levels are meaningless. Thus, in this paper, we consider the case where $\alpha, \beta$ are in the interval $[0.5,1]$.

Theorem 5. Assume that $\eta \sim \mathcal{N}(\mu, \Sigma)$, where $\Sigma$ is deterministic matrix, $\mu_{i}, i=1,2, \ldots, n$, are mutual independent fuzzy variables, and $\mu^{T} x$ is continuous. In the case of $\alpha, \beta \geq 0.5$, the following results hold.

(i) $\operatorname{Cr}\left\{\mu^{T} x \geq \Phi^{-1}(\alpha) \sqrt{x^{T} D D^{T} x}+z\right\} \geq \beta \Leftrightarrow$ $\Phi^{-1}(\alpha) \sqrt{x^{T} D D^{T} x}+z-\left(\mu^{T} x\right)_{\text {sup }}(\beta) \leq 0$.

(ii) $\mathscr{C}=\left\{x \mid \operatorname{Cr}\left\{\mu^{T} x \geq \Phi^{-1}(\alpha) \sqrt{x^{T} D D^{T} x}+z\right\} \geq \beta\right\}$ is a convex set.

Proof. We first prove the necessity of assertion (i). By the definition of the optimistic value of fuzzy variable $\mu^{T} x$, one has

$$
\left(\mu^{T} x\right)_{\text {sup }}(\beta) \geq \Phi^{-1}(\alpha) \sqrt{x^{T} D D^{T} x}+z,
$$

which implies

$$
\Phi^{-1}(\alpha) \sqrt{x^{T} D D^{T} x}+z-\left(\mu^{T} x\right)_{\text {sup }}(\beta) \leq 0 .
$$

The necessity of assertion (i) holds true.

We next prove the sufficiency of assertion (i). According to the definition of optimistic value, we know the credibilistic constraint $\operatorname{Cr}\left\{\mu^{T} x \geq\left(\mu^{T} x\right)_{\text {sup }}(\beta)\right\} \geq \beta$ holds. Since

$$
\left(\mu^{T} x\right)_{\sup }(\beta) \geq \Phi^{-1}(\alpha) \sqrt{x^{T} D D^{T} x}+z
$$

is equivalent to

$$
\Phi^{-1}(\alpha) \sqrt{x^{T} D D^{T} x}+z-\left(\mu^{T} x\right)_{\text {sup }}(\beta) \leq 0,
$$

we have

$$
\operatorname{Cr}\left\{\mu^{T} x \geq \Phi^{-1}(\alpha) \sqrt{x^{T} D D^{T} x}+z\right\} \geq \beta
$$

due to the continuity of $\mu^{T} x$. The sufficiency is proved.

In what follows, we prove assertion (ii).

According to the property of optimistic value, the equality $\left(\mu^{T} x\right)_{\text {sup }}(\beta)=\sum_{i=1}^{n} x_{i} \mu_{i \text {,sup }}(\beta)$ holds. According to assertion (i), the set $\mathscr{C}$ is equivalent to

$$
\left\{x \mid \Phi^{-1}(\alpha) \sqrt{x^{T} D D^{T} x}+z-\sum_{i=1}^{n} x_{i} \mu_{i, \mathrm{sup}}(\beta) \leq 0\right\} .
$$

It is easy to know that $\Phi^{-1}(\alpha) \geq 0$ for $\alpha \geq 0.5, \sqrt{x^{T} D D^{T} x}$ is a convex function with respect to $x$, and $\sum_{i=1}^{n} x_{i} \mu_{i \text {,sup }}(\beta)$ is a linear function with respect to $x_{i}$. According to the properties of convex function, $\left\{x \mid \Phi^{-1}(\alpha) \sqrt{x^{T} D D^{T} x}+z-\right.$ $\left.\sum_{i=1}^{n} x_{i} \mu_{i \text {,sup }}(\beta) \leq 0\right\}$ is a convex set. The proof of assertion (ii) is complete. 
Based on the above theoretical analysis, the following theorem gives the deterministic equivalent model of model (20).

Theorem 6. Assume that $\eta \sim \mathcal{N}(\mu, \Sigma)$, where $\Sigma$ is a deterministic matrix, $\mu_{i}, i=1,2, \ldots, n$, are mutual independent fuzzy variables, and $\mu^{T} x$ is continuous. Then, in the case of $\alpha, \beta \geq$ $0.5, E V$-ERV model (20) is equivalent to the following programming model:

$$
\begin{array}{ll}
\max & \sum_{i=1}^{n} x_{i} E\left[\mu_{i}\right] \\
\text { s.t. } & \Phi^{-1}(\alpha) \sqrt{x^{T} D D^{T} x}+z-\sum_{i=1}^{n} x_{i} \mu_{i, \text { sup }}(\beta) \leq 0 \\
& z \geq \kappa \\
& \sum_{i=1}^{n} x_{i}=1 \\
& x_{i} \geq 0, i=1,2, \ldots, n .
\end{array}
$$

On the basis of Theorems 5 and 6 , we arrive at the following result.

Theorem 7. Assume that $\eta \sim \mathcal{N}(\mu, \Sigma)$, where $\Sigma$ is a deterministic matrix, $\mu_{i}, i=1,2, \ldots, n$, are mutual independent fuzzy variables, and $\mu^{T} x$ is continuous. Then, in the case of $\alpha$, $\beta \geq 0.5$, model (27) is a convex programming model.

Proof. In the case of $\alpha, \beta \geq 0.5$, by Theorem $5, \mathscr{C}=\{x \mid$ $\left.\operatorname{Cr}\left\{\mu^{T} x \geq \Phi^{-1}(\alpha) \sqrt{x^{T} D D^{T} x}+z\right\} \geq \beta\right\}$ is a convex set. Then the feasible region is convex. Due to the linearity of the objective function and the convexity of the feasible region, model (27) is a convex programming model.

\section{Equivalent Deterministic Convex Programming Models}

In this section, we specify the possibility distributions of fuzzy parameters $\mu_{i}, i=1,2, \ldots, n$, so that the analytical expressions of $E\left[\mu_{i}\right]$ and $\mu_{i \text {,sup }}(\beta)$ are available. We consider the cases that fuzzy parameters $\mu_{i}, i=1,2, \ldots, n$, are characterized by trapezoidal, triangular, and normal fuzzy variables, respectively.

Case 1 (trapezoidal fuzzy variables). Let $\eta \sim \mathcal{N}(\mu, \Sigma), \mu_{i}=$ $\left(r_{i}^{(1)}, r_{i}^{(2)}, r_{i}^{(3)}, r_{i}^{(4)}\right), i=1,2, \ldots, n$, be mutual independent trapezoidal fuzzy variables. According to [34], the expected return of $\mu^{T} x$ is

$$
\begin{aligned}
& E\left[\mu^{T} x\right] \\
& \quad=\frac{1}{4}\left(\sum_{i=1}^{n} r_{i}^{(1)} x_{i}+\sum_{i=1}^{n} r_{i}^{(2)} x_{i}+\sum_{i=1}^{n} r_{i}^{(3)} x_{i}+\sum_{i=1}^{n} r_{i}^{(4)} x_{i}\right) .
\end{aligned}
$$

The optimistic value of $\mu^{T} x$ is

$$
\begin{aligned}
\sum_{i=1}^{n} x_{i} \mu_{i, \text { sup }}(\beta)= & 2(1-\beta) \sum_{i=1}^{n} r_{i}^{(2)} x_{i} \\
& +(2 \beta-1) \sum_{i=1}^{n} r_{i}^{(1)} x_{i}
\end{aligned}
$$

where $\beta \geq 0.5$.

As a consequence, for any given parameters $\alpha, \beta \geq 0.5$, by Theorem 6, model (27) is equivalent to the following deterministic convex programming model:

$$
\begin{aligned}
\max & \frac{1}{4}\left(\sum_{i=1}^{n} r_{i}^{(1)} x_{i}+\sum_{i=1}^{n} r_{i}^{(2)} x_{i}+\sum_{i=1}^{n} r_{i}^{(3)} x_{i}+\sum_{i=1}^{n} r_{i}^{(4)} x_{i}\right) \\
\text { s.t. } \quad \Phi^{-1}(\alpha) \sqrt{x^{T} D D^{T} x}+z & \\
& -2(1-\beta) \sum_{i=1}^{n} r_{i}^{(2)} x_{i}-(2 \beta-1) \sum_{i=1}^{n} r_{i}^{(1)} x_{i} \leq 0
\end{aligned}
$$

$$
\begin{aligned}
& z \geq \kappa \\
& \sum_{i=1}^{n} x_{i}=1 \\
& x_{i} \geq 0, \quad i=1,2, \ldots, n .
\end{aligned}
$$

Triangular fuzzy variable is a special case of trapezoidal fuzzy variable. The following remark is about the result related to triangular possibility distributions.

Remark 8. Let $\eta \sim \mathcal{N}(\mu, \Sigma), \mu_{i}=\left(r_{i}^{(1)}, r_{i}^{(2)}, r_{i}^{(3)}\right), i=$ $1,2, \ldots, n$, be mutual independent triangular fuzzy variables. According to model (30), for any given parameters $\alpha, \beta \geq 0.5$, the equivalent deterministic convex programming model of model (27) is

$$
\begin{array}{ll}
\max & \frac{1}{4}\left(\sum_{i=1}^{n} r_{i}^{(1)} x_{i}+2 \sum_{i=1}^{n} r_{i}^{(2)} x_{i}+\sum_{i=1}^{n} r_{i}^{(3)} x_{i}\right) \\
\text { s.t. } & \Phi^{-1}(\alpha) \sqrt{x^{T} D D^{T} x}+z-2(1-\beta) \sum_{i=1}^{n} r_{i}^{(2)} x_{i} \\
& -(2 \beta-1) \sum_{i=1}^{n} r_{i}^{(1)} x_{i} \leq 0
\end{array}
$$

$$
\begin{aligned}
& z \geq \kappa \\
& \sum_{i=1}^{n} x_{i}=1 \\
& x_{i} \geq 0, \quad i=1,2, \ldots, n .
\end{aligned}
$$

Case 2 (normal fuzzy variables). Let $\eta \sim \mathcal{N}(\mu, \Sigma), \mu_{i}=n\left(m_{i}\right.$, $\left.\sigma_{i}\right), i=1,2, \ldots, n$, be mutual independent normal fuzzy 
variables. According to [34], the expected return of $\mu^{T} x$ is $E\left[\mu^{T} x\right]=\sum_{i=1}^{n} m_{i} x_{i}$. The optimistic value of $\mu^{T} x$ is

$$
\sum_{i=1}^{n} x_{i} \mu_{i, \text { sup }}(\beta)=\sum_{i=1}^{n} m_{i} x_{i}+\sqrt{-2 \ln 2(1-\beta)} \sum_{i=1}^{n} x_{i}^{2} \sigma_{i},
$$

where $\beta \geq 0.5$.

Therefore, according to Theorem 6 , for any given parameters $\alpha, \beta \geq 0.5$, the equivalent deterministic convex programming model of model (27) reads

$$
\begin{array}{ll}
\max & \sum_{i=1}^{n} m_{i} x_{i} \\
\text { s.t. } & \Phi^{-1}(\alpha) \sqrt{x^{T} D D^{T} x}+z-\sum_{i=1}^{n} m_{i} x_{i} \\
& -\sqrt{-2 \ln 2(1-\beta)} \sum_{i=1}^{n} x_{i}^{2} \sigma_{i} \leq 0 \\
& z \geq \kappa
\end{array}
$$

$$
\begin{aligned}
& \sum_{i=1}^{n} x_{i}=1 \\
& x_{i} \geq 0, i=1,2, \ldots, n .
\end{aligned}
$$

\section{Numerical Experiments and Comparison Study}

In this section, we will conduct some numerical experiments to demonstrate the feasibility and effectiveness of the developed equilibrium optimization method. We first give some descriptions about our portfolio selection problem in the next subsection.

5.1. Problem Description. Assume that there are 20 potential risky assets for an investor. In this portfolio selection problem, the return rates have twofold uncertainty and are represented by random fuzzy variables. For the sake of presentation, we suppose that the prescribed confidence levels $\alpha, \beta$ take their values from the interval $[0.5,1]$.

Let $\eta=\left(\eta_{1}, \eta_{2}, \ldots, \eta_{20}\right) \sim \mathcal{N}(\mu, \Sigma)$ and $\mu_{i}=\left(r_{i}^{(1)}, r_{i}^{(2)}\right.$, $\left.r_{i}^{(3)}, r_{i}^{(4)}\right), i=1,2, \ldots, 20$, be mutual independent trapezoidal fuzzy variables. The possibility distributions of $\mu_{i}, i=1,2$, $\ldots, 20$, are provided in Table 1 , and the covariance matrix $\Sigma=$ $\left(\sigma_{i j}\right)_{20 \times 20}$ is expressed as $10^{-2}\left(\Sigma_{1} \Sigma_{2}\right)$, where the matrices $\Sigma_{1}$ and $\Sigma_{2}$ are defined as follows:

$$
\Sigma_{1}=\left(\begin{array}{cccccccccc}
0.6198 & 0.1155 & 0.1096 & -0.0685 & 0.0038 & 0.1222 & 0.0049 & -0.0770 & 0.0319 & -0.0368 \\
0.1155 & 0.5989 & -0.0937 & 0.0757 & 0.0531 & 0.1086 & -0.0041 & 0.0598 & 0.1067 & 0.0351 \\
0.1096 & -0.0937 & 0.6824 & 0.0215 & 0.0389 & -0.0539 & -0.0004 & 0.0912 & -0.0550 & -0.0010 \\
-0.0685 & 0.0757 & 0.0215 & 0.6481 & 0.0718 & 0.0130 & 0.1047 & -0.0651 & -0.0494 & -0.0673 \\
0.0038 & 0.0531 & 0.0389 & 0.0718 & 0.6583 & -0.1011 & -0.0604 & -0.0524 & -0.0097 & 0.0441 \\
0.1222 & 0.1086 & -0.0539 & 0.0130 & -0.1011 & 0.5062 & 0.0014 & -0.0576 & -0.0785 & -0.0182 \\
0.0049 & -0.0041 & -0.0004 & 0.1047 & -0.0604 & 0.0014 & 0.5376 & -0.0959 & -0.0997 & -0.0202 \\
-0.0770 & 0.0598 & 0.0912 & -0.0651 & -0.0524 & -0.0576 & -0.0959 & 0.7562 & 0.0317 & -0.0862 \\
0.0319 & 0.1067 & -0.0550 & -0.0494 & -0.0097 & -0.0785 & -0.0997 & 0.0317 & 0.5243 & -0.0096 \\
-0.0368 & 0.0351 & -0.0010 & -0.0673 & 0.0441 & -0.0182 & -0.0202 & -0.0862 & -0.0096 & 0.8199 \\
0.0536 & -0.0566 & 0.0058 & -0.0055 & -0.0926 & 0.1329 & -0.1258 & -0.0200 & -0.1145 & 0.1264 \\
0.0596 & -0.0767 & 0.0744 & -0.0118 & -0.0230 & -0.0668 & -0.1022 & -0.1742 & 0.0360 & -0.0647 \\
0.0506 & -0.1416 & -0.1521 & 0.0506 & 0.0718 & -0.0597 & -0.0279 & -0.0599 & 0.1710 & 0.0026 \\
0.0278 & 0.0399 & -0.1190 & 0.1091 & -0.0933 & -0.0491 & -0.0045 & 0.0752 & -0.0981 & 0.0230 \\
0.1505 & -0.0353 & 0.0332 & -0.0742 & -0.0080 & -0.0379 & 0.0743 & 0.0630 & 0.0547 & 0.1186 \\
0.0356 & -0.0105 & 0.0371 & -0.0214 & 0.0032 & -0.1460 & -0.0696 & -0.0514 & 0.0008 & -0.0655 \\
0.0969 & -0.0331 & -0.1452 & 0.0123 & 0.0205 & -0.0958 & 0.0567 & 0.0483 & -0.0491 & -0.0479 \\
-0.0039 & -0.0670 & -0.0077 & -0.0698 & -0.0421 & -0.0514 & 0.1034 & 0.0345 & 0.0241 & 0.0215 \\
-0.0289 & -0.0619 & 0.0332 & -0.0900 & -0.0145 & -0.0699 & -0.1032 & -0.0703 & -0.0612 & 0.0326 \\
0.0825 & 0.0193 & 0.0145 & 0.0466 & 0.1161 & 0.1231 & -0.0721 & 0.0214 & 0.0432 & 0.0527
\end{array}\right),
$$




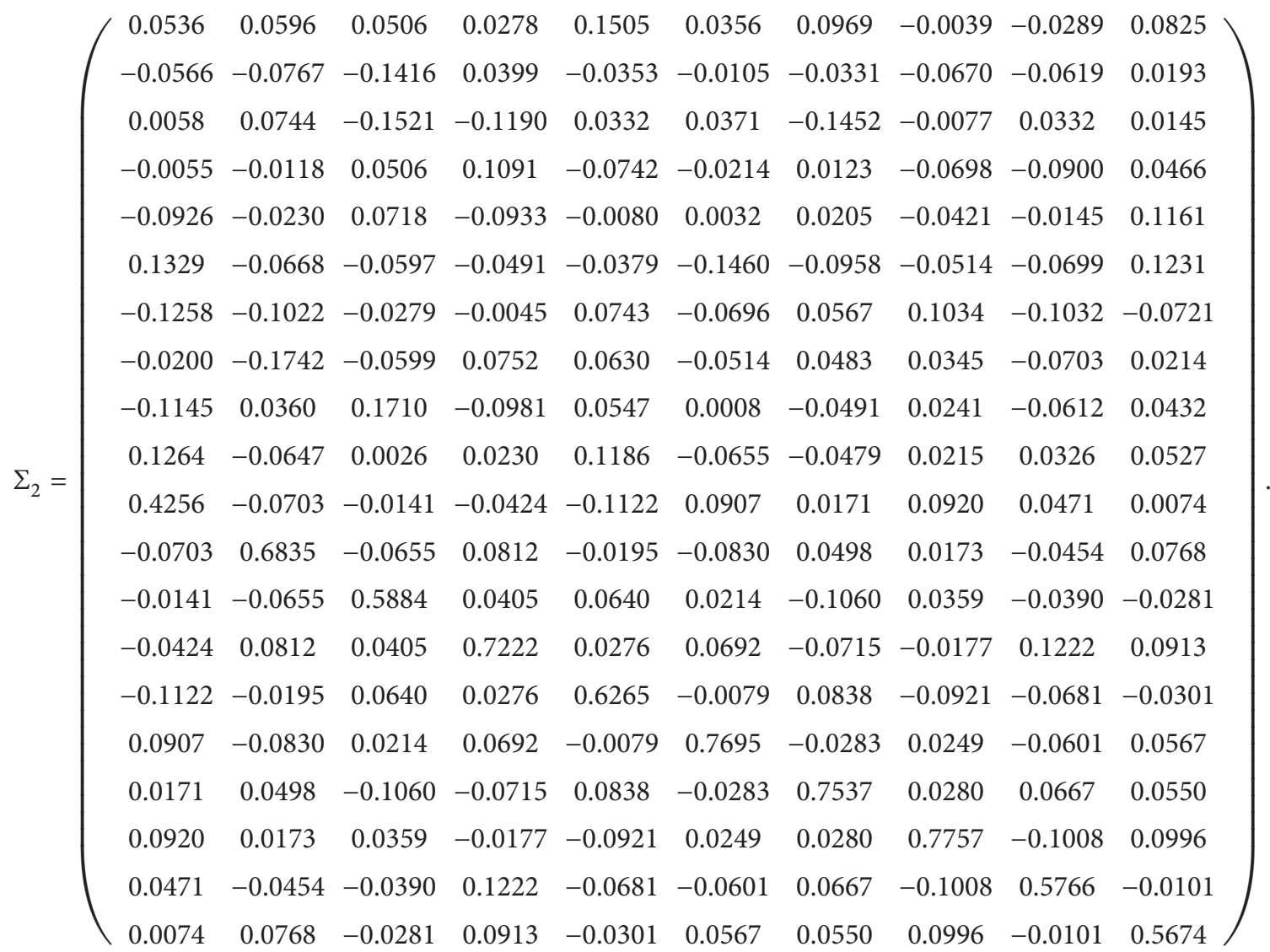

In this case, model (30) becomes the following deterministic convex programming model:

$$
\begin{aligned}
& \max \quad 0.03175 x_{1}+0.0315 x_{2}+0.03325 x_{3} \\
&+ 0.03325 x_{4}+0.0315 x_{5}+0.0345 x_{6} \\
&+0.03175 x_{7}+0.03175 x_{8}+0.0325 x_{9} \\
&+0.03225 x_{10}+0.0315 x_{11}+0.03125 x_{12} \\
&+0.033 x_{13}+0.03325 x_{14}+0.03125 x_{15} \\
&+0.03425 x_{16}+0.03175 x_{17}+0.0315 x_{18} \\
&+0.03225 x_{19}+0.031975 x_{20} \\
& \text { s.t. } \quad 10^{-1} \Phi^{-1}(\alpha)\left(\sum_{i=1}^{20} \sum_{j=1}^{20} \sigma_{i j} x_{i} x_{j}\right)^{1 / 2}+z \\
& \quad-2(1-\beta) V_{2}(x)-(2 \beta-1) V_{1}(x) \leq 0 \\
& \quad z \geq \\
& \sum_{i=1}^{20} x_{i}=1 \\
& x_{i} \geq 0, i=1,2, \ldots, 20,
\end{aligned}
$$

where the coefficient of $x_{i}$ in the objective function is the expected value of trapezoidal fuzzy variable $\mu_{i}, \sigma_{i j}$ in the ERV constraint is the element of matrix $\Sigma$ at the $i$ th row and the $j$ th column, and the analytical expressions of functions $V_{1}(x)$ and $V_{2}(x)$ in the ERV constraint are defined as follows:

$$
\begin{aligned}
V_{1}(x)= & \sum_{i=1}^{20} r_{i}^{(1)} x_{i} \\
= & 0.005 x_{1}+0.006 x_{2}+0.007 x_{3}+0.004 x_{4} \\
& +0.005 x_{5}+0.006 x_{6}+0.004 x_{7}+0.005 x_{8} \\
& +0.006 x_{9}+0.005 x_{10}+0.004 x_{11} \\
& +0.005 x_{12}+0.006 x_{13}+0.004 x_{14} \\
& +0.004 x_{15}+0.005 x_{16}+0.004 x_{17} \\
& +0.004 x_{18}+0.005 x_{19}+0.004 x_{20} \\
& 20 \\
V_{2}(x)= & \sum_{i=1}^{(2)} r_{i} \\
= & 0.036 x_{1}+0.037 x_{2}+0.039 x_{3}+0.038 x_{4} \\
& +0.037 x_{5}+0.041 x_{6}+0.036 x_{7}+0.038 x_{8} \\
& +0.038 x_{9}+0.038 x_{10}+0.036 x_{11}
\end{aligned}
$$


TABLE 1: The distributions of trapezoidal fuzzy parameters $\mu_{i}$.

\begin{tabular}{lc}
\hline Optional risky assets & Possibility distributions \\
\hline 1 & $\mu_{1}=(0.005,0.036,0.038,0.048)$ \\
2 & $\mu_{2}=(0.006,0.037,0.038,0.045)$ \\
3 & $\mu_{3}=(0.007,0.039,0.040,0.047)$ \\
4 & $\mu_{4}=(0.004,0.038,0.040,0.051)$ \\
5 & $\mu_{5}=(0.005,0.037,0.039,0.045)$ \\
6 & $\mu_{6}=(0.006,0.041,0.042,0.049)$ \\
7 & $\mu_{7}=(0.004,0.036,0.039,0.048)$ \\
8 & $\mu_{8}=(0.005,0.038,0.039,0.045)$ \\
9 & $\mu_{9}=(0.006,0.038,0.040,0.046)$ \\
10 & $\mu_{10}=(0.005,0.038,0.040,0.046)$ \\
11 & $\mu_{11}=(0.004,0.036,0.039,0.047)$ \\
12 & $\mu_{12}=(0.005,0.037,0.0385,0.0445)$ \\
13 & $\mu_{13}=(0.006,0.039,0.0405,0.0465)$ \\
14 & $\mu_{14}=(0.004,0.038,0.041,0.050)$ \\
15 & $\mu_{15}=(0.004,0.037,0.0392,0.0448)$ \\
16 & $\mu_{16}=(0.005,0.041,0.0425,0.0485)$ \\
17 & $\mu_{17}=(0.004,0.036,0.040,0.047)$ \\
18 & $\mu_{18}=(0.004,0.038,0.0385,0.0455)$ \\
19 & $\mu_{19}=(0.005,0.038,0.0395,0.0465)$ \\
20 & $\mu_{20}=(0.004,0.038,0.0395,0.0464)$ \\
\hline
\end{tabular}

TABLE 2: Computational results with $\alpha=0.8, \beta=0.8$, and $\kappa=0.006$.

\begin{tabular}{lccccc}
\hline \multirow{4}{*}{ Investment ratios } & $x_{1}$ & $x_{2}$ & $x_{3}$ & $x_{4}$ & $x_{5}$ \\
& $x_{6}$ & $x_{7}$ & $x_{8}$ & $x_{9}$ & $x_{10}$ \\
& $x_{11}$ & $x_{12}$ & $x_{13}$ & $x_{14}$ & $x_{15}$ \\
& $x_{16}$ & $x_{17}$ & $x_{18}$ & $x_{19}$ & $x_{20}$ \\
\hline \multirow{4}{*}{ Values } & 0 & 0 & 0.06599 & 0.03981 & 0.03508 \\
& 0.19412 & 0.07107 & 0.06188 & 0.07594 & 0.04273 \\
& 0 & 0.05974 & 0.07214 & 0.02827 & 0 \\
& 0.11136 & 0.04525 & 0.00917 & 0.08748 & 0 \\
\hline
\end{tabular}

$$
\begin{aligned}
& +0.037 x_{12}+0.039 x_{13}+0.038 x_{14} \\
& +0.037 x_{15}+0.041 x_{16}+0.036 x_{17} \\
& +0.038 x_{18}+0.038 x_{19}+0.038 x_{20}
\end{aligned}
$$

5.2. Computational Results. Firstly, we set the confidence levels $\alpha=0.8, \beta=0.8$ and the prescribed value of ERV $\kappa=0.006$ and solve model (35) by LINGO software. After 31 iterations, we obtain the distributive investment ratios among 20 risky assets, which are reported in Table 2 . Due to the convexity of model (35), the obtained investment scheme is the global optimal solution, and its corresponding optimal objective value is 0.03293 .

Secondly, considering that investors may have different attitudes towards risk, model (35) is solved under various values of parameters $\alpha, \beta$, and $\kappa$. The obtained computational results are provided in Table 3 , from which we observe the effect of model parameters on the solution quality of the EV-ERV model. The computational results demonstrate that

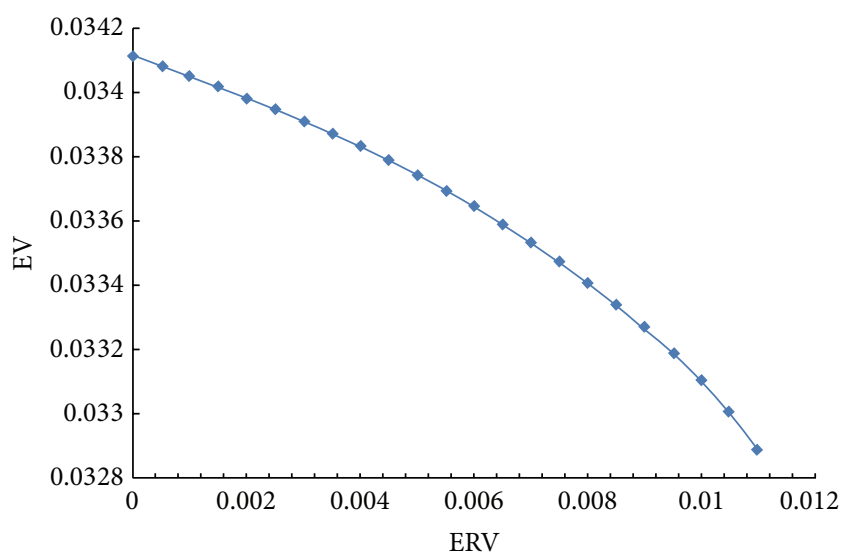

FIgURE 1: Relationship between ERV and EV under $\alpha=0.7$ and $\beta=0.8$.

when the values of parameters $\alpha, \beta$, and $\kappa$ are changed, the investment ratios and objective values are also changed accordingly. If we fix two of the parameters $\alpha, \beta$, and $\kappa$, the objective value will decrease with respect to the remaining parameter. For example, when $\alpha=0.8$ and $\kappa=0.006$, the objective values are EV $=0.03329,0.03302,0.03261$, corresponding to $\beta=0.75,0.79,0.82$, respectively. In addition, the value of $z$ is always equal to that of $\kappa$; that is, the ERV is attainable and equal to $\kappa$, which is evident from theoretical analysis.

Thirdly, we demonstrate the relationship between ERV and $\mathrm{EV}$ in our equilibrium optimization model via numerical experiments. For this purpose, we set the confidence levels $\alpha=0.7$ and $\beta=0.8$. The computational results are plotted in Figure 1, from which we observe that all the points under the line satisfy the constraints in our problem, and are called feasible solutions. The points on the line are the optimal solutions for the given $\alpha$ and $\beta$. The area under the line is the effective coverage.

5.3. Comparing with Stochastic Optimization Method. In this subsection, we compare the proposed equilibrium optimization method with classical stochastic optimization method. For the sake of comparison, we also employ the data provided in Section 5.1, and replace the trapezoidal fuzzy variables in Table 1 by their expected values. As a result, the return rate of each asset is characterized by random variable. In this case, the random fuzzy return vector $\eta$ degenerates into a random return vector $\eta \sim \mathcal{N}(\mu, \Sigma)$, where $\mu, \Sigma$ are deterministic vector and matrix. That is, the return rates $\eta_{i}$, $i=1,2, \ldots, 20$, are considered as normal random variables, and their dependence are characterized by covariance matrix $\sum$.

By calculation, the expected return rate vector is

$\mu=(0.03175,0.0315,0.03325,0.03325,0.0315$,

$0.0345,0.03175,0.03175,0.0325,0.03225,0.0315$,

$0.03125,0.033,0.03325,0.03125,0.03425$, 
TABLE 3: The optimal solutions of equilibrium optimization model under various values of parameters.

\begin{tabular}{|c|c|c|c|c|c|c|c|c|}
\hline \multicolumn{3}{|c|}{ Parameters } & \multicolumn{5}{|c|}{ Investment ratios } & \multirow[t]{2}{*}{ Objective values } \\
\hline \multirow{4}{*}{$\alpha$} & \multirow{4}{*}{$\beta$} & \multirow{4}{*}{$\kappa$} & $x_{1}$ & $x_{2}$ & $x_{3}$ & $x_{4}$ & $x_{5}$ & \\
\hline & & & $x_{6}$ & $x_{7}$ & $x_{8}$ & $x_{9}$ & $x_{10}$ & \multirow[t]{3}{*}{$\mathrm{EV}$} \\
\hline & & & $x_{11}$ & $x_{12}$ & $x_{13}$ & $x_{14}$ & $x_{15}$ & \\
\hline & & & $x_{16}$ & $x_{17}$ & $x_{18}$ & $x_{19}$ & $x_{20}$ & \\
\hline \multirow{4}{*}{0.78} & \multirow{4}{*}{0.8} & \multirow{4}{*}{0.006} & 0 & 0 & 0.08332 & 0.04127 & 0.02539 & \multirow{4}{*}{0.03308} \\
\hline & & & 0.21540 & 0.05473 & 0.04693 & 0.07931 & 0.03955 & \\
\hline & & & 0 & 0.04148 & 0.07848 & 0.04074 & 0 & \\
\hline & & & 0.12020 & 0.05187 & 0.00402 & 0.07731 & 0 & \\
\hline \multirow{4}{*}{0.8} & \multirow{4}{*}{0.8} & \multirow{4}{*}{0.006} & 0 & 0 & 0.06599 & 0.03981 & 0.03508 & \multirow{4}{*}{0.03293} \\
\hline & & & 0.19412 & 0.07107 & 0.06188 & 0.07594 & 0.04273 & \\
\hline & & & 0 & 0.05974 & 0.07214 & 0.02827 & 0 & \\
\hline & & & 0.11136 & 0.04525 & 0.00917 & 0.08748 & 0 & \\
\hline \multirow{4}{*}{0.82} & \multirow{4}{*}{0.8} & \multirow{4}{*}{0.006} & 0 & 0.01039 & 0.04343 & 0.03564 & 0.04671 & \multirow{4}{*}{0.03271} \\
\hline & & & 0.16033 & 0.09309 & 0.08176 & 0.06673 & 0.04612 & \\
\hline & & & 0.00108 & 0.08616 & 0.06753 & 0.00916 & 0 & \\
\hline & & & 0.09753 & 0.03613 & 0.01668 & 0.10153 & 0 & \\
\hline \multirow{4}{*}{0.8} & \multirow{4}{*}{0.75} & \multirow{4}{*}{0.006} & 0 & 0 & 0.10802 & 0.04491 & 0.01061 & \multirow{4}{*}{0.03329} \\
\hline & & & 0.24738 & 0.02916 & 0.02402 & 0.08360 & 0.03457 & \\
\hline & & & 0 & 0.01334 & 0.08674 & 0.05983 & 0 & \\
\hline & & & 0.13391 & 0.06153 & 0 & 0.06240 & 0 & \\
\hline \multirow{4}{*}{0.8} & & & 0 & 0 & 0.07626 & 0.04095 & 0.02916 & \\
\hline & 0.79 & 0.006 & 0.20703 & 0.06120 & 0.05276 & 0.07790 & 0.04081 & 0.03302 \\
\hline & & & 0 & 0.04857 & 0.07578 & 0.03599 & 0 & \\
\hline & & & 0.11685 & 0.04931 & 0.06131 & 0.08131 & 0 & \\
\hline & & & 0 & 0.01762 & 0.03796 & 0.03247 & 0.05033 & \\
\hline 08 & 082 & 0006 & 0.14456 & 0.09980 & 0.08373 & 0.06536 & 0.04170 & 003261 \\
\hline 0.0 & 0.02 & 0.000 & 0.01818 & 0.09175 & 0.06540 & 0.00562 & 0.00613 & 0.05201 \\
\hline & & & 0.08971 & 0.03125 & 0.01620 & 0.10224 & 0 & \\
\hline & & & 0 & 0 & 0.10076 & 0.04330 & 0.01526 & \\
\hline 0.78 & 0.78 & 0.006 & 0.23748 & 0.03741 & 0.03131 & 0.08249 & 0.03619 & 0.03323 \\
\hline & & & 0 & 0.02234 & 0.08461 & 0.05375 & 0 & \\
\hline & & & 0.12957 & 0.05859 & 0 & 0.06693 & 0 & \\
\hline & & & 0 & 0 & 0.07275 & 0.04044 & 0.03112 & \\
\hline 078 & 078 & 0008 & 0.20291 & 0.06426 & 0.05606 & 0.07705 & 0.04148 & 003299 \\
\hline 0.10 & 0.10 & 0.000 & 0 & 0.05253 & 0.07474 & 0.03300 & 0 & 0.05299 \\
\hline & & & 0.11520 & 0.04764 & 0.00727 & 0.08355 & 0 & \\
\hline & & & 0 & 0 & 0.05231 & 0.03833 & 0.04272 & \\
\hline 078 & 078 & 0.009 & 0.17766 & 0.08354 & 0.07411 & 0.07299 & 0.04528 & 003282 \\
\hline & & & 0 & 0.07457 & 0.06753 & 0.01768 & 0 & \\
\hline & & & 0.10462 & 0.03948 & 0.01339 & 0.09579 & 0 & \\
\hline
\end{tabular}

The objective function is $E\left[\eta^{T} x\right]=\sum_{i=1}^{n} x_{i} \mu_{i}$, and the ERV constraint is expressed as

$$
\Phi^{-1}(\alpha) \sqrt{x^{T} \Sigma x}+z-\mu^{T} x \leq 0 .
$$

As a consequence, our equilibrium portfolio optimization problem reduces to the following convex programming model:

$$
\begin{aligned}
\max & 0.03175 x_{1}+0.0315 x_{2}+0.03325 x_{3}+0.03325 x_{4} \\
& +0.0315 x_{5}+0.0345 x_{6}+0.03175 x_{7}+0.03175 x_{8}
\end{aligned}
$$

$$
\begin{aligned}
& +0.0325 x_{9}+0.03225 x_{10}+0.0315 x_{11}+0.03125 x_{12} \\
& +0.033 x_{13}+0.03325 x_{14}+0.03125 x_{15}+0.03425 x_{16} \\
& +0.03175 x_{17}+0.0315 x_{18} \\
& +0.03225 x_{19}+0.031975 x_{20}
\end{aligned}
$$

s.t. $10^{-1} \Phi^{-1}(\alpha)\left(\sum_{i=1}^{20} \sum_{j=1}^{20} \sigma_{i j} x_{i} x_{j}\right)^{1 / 2}$ 
TABLE 4: Computational results with $\alpha=0.8$ and $\kappa=0.006$.

\begin{tabular}{lccccc}
\hline \multirow{4}{*}{ Investment ratios } & $x_{1}$ & $x_{2}$ & $x_{3}$ & $x_{4}$ & $x_{5}$ \\
& $x_{6}$ & $x_{7}$ & $x_{8}$ & $x_{9}$ & $x_{10}$ \\
& $x_{11}$ & $x_{12}$ & $x_{13}$ & $x_{14}$ & $x_{15}$ \\
& $x_{16}$ & $x_{17}$ & $x_{18}$ & $x_{19}$ & $x_{20}$ \\
\hline \multirow{4}{*}{ Values } & 0 & 0 & 0.11689 & 0.04596 & 0 \\
& 0.41539 & 0 & 0 & 0.01806 & 0 \\
& 0 & 0 & 0.08538 & 0.07634 & 0 \\
& 0.24198 & 0 & 0 & 0 & 0 \\
\hline
\end{tabular}

$$
\begin{aligned}
& +z-\left(0.03175 x_{1}+0.0315 x_{2}\right. \\
& +0.03325 x_{3}+0.03325 x_{4}+0.0315 x_{5}+0.0345 x_{6} \\
& +0.03175 x_{7}+0.03175 x_{8}+0.0325 x_{9}+0.03225 x_{10} \\
& +0.0315 x_{11}+0.03125 x_{12}+0.033 x_{13} \\
& +0.03325 x_{14}+0.03125 x_{15}+0.03425 x_{16}+0.03175 x_{17} \\
& \left.+0.0315 x_{18}+0.03225 x_{19}+0.031975 x_{20}\right) \leq 0 \\
& z \geq \kappa \\
& \sum_{i=1}^{20} x_{i}=1 \\
& x_{i} \geq 0, i=1,2, \ldots, 20
\end{aligned}
$$

To solve model (39) by LINGO software, we set the confidence level $\alpha=0.8$ and the prescribed value of ERV $\kappa=0.006$. The obtained optimal portfolio is collected in Table 4 , with the optimal objective value 0.03398 .

To identify the influences of model parameters on solution quality, the stochastic model (39) is also solved under various values of parameters $\alpha$ and $\kappa$. The optimal investment solutions are reported in Table 5, from which we find that the solution results are different under various values of $\alpha$ and $\kappa$. If we set parameter $\kappa=0.006$, then the objective function is decreasing with respect to confidence level $\alpha$. Given the parameter $\alpha$, the objective function is also decreasing with respect to parameter $\kappa$.

We now compare the computational results reported in Tables 2 and 4 as well as their optimal objective values. When model parameters are set to the same values, our equilibrium optimization method and stochastic optimization method produce different investment schemes. The equilibrium method selects 15 risky assets, while stochastic method selects 7 risky assets. In addition, two optimization methods suggest different investment ratios for the same risky asset. For example, the equilibrium method provides investment ratio 0.06599 for the third risky asset, while the stochastic method provides investment ratio $x_{3}=0.11689$. From the viewpoint of distributive investment, we conclude that our equilibrium method is superior to the classical stochastic method.

We continue to compare the computational results reported in Tables 3 and 5, from which we observe that the equilibrium method and stochastic method provide different combinations of risky assets and different optimal return rates. For example, when we set probability level $\alpha=$ 0.82 , credibility level $\beta=0.8$, and $\kappa=0.006$, the equilibrium method provides the following optimal investment plan:

$$
\begin{aligned}
x= & (0,0.01039,0.04343,0.03564,0.04671,0.16033, \\
& 0.09309,0.08176,0.06673,0.04612,0.00108, \\
& 0.08616,0.06753,0.00916,0,0.09753,0.03613, \\
& 0.01668,0.10153,0)^{T},
\end{aligned}
$$

whose objective value is 0.03271 . The stochastic method only selects 9 risky assets $i, i=3,4,6,9,13,14,16,17,19$. The corresponding investment ratios are $0.12286,0.04955,0.37902$, $0.04157,0.08867,0.08131,0.21614,0.01161$, and 0.00927 , respectively. The optimal return rate provided by the stochastic method is 0.03386 .

On the other hand, under the same values of model parameters, we observe that the optimal objective values in Table 5 are larger than those provided in Table 3. For example, when $\alpha=0.78, \beta=0.8$, and $\kappa=0.006$, the equilibrium method provides the optimal value 0.03308 , while stochastic method provides the optimal value 0.03408 . This is because the credibilistic constraints are relaxed when the random fuzzy return rates degenerate into random ones. Therefore, we may obtain higher expected return rate. Nevertheless, the superiority of our equilibrium method is reflected in terms of diversification.

Finally, we want to point out that an investor may often encounter a hybrid uncertain environment in modern financial markets. In this situation, the investor cannot ignore the influence of fuzzy uncertainty on the solution quality. The computational results support our arguments. For example, when probability level $\alpha=0.78$ and $\kappa=0.006$, the stochastic model (39) suggests the optimal investment scheme $x^{*}=(0,0,0.09906,0.03658,0,0.46099,0,0,0,0,0,0,0.0670$, $0.06212,0,0.27424,0,0,0,0)^{T}$. However, $x^{*}$ is not the feasible solution to equilibrium optimization model (35) with probability level $\alpha=0.78$, credibility level $\beta=0.78$, and $\kappa=0.006$. In order to keep $x^{*}$ feasible to model (35), we have to reduce the value of parameter $\kappa$ from 0.006 to -0.00717 . By the definition of $\kappa$, the optimal solution corresponding to -0.00717 is meaningless from a practical viewpoint.

As a consequence, we conclude from the computational results that our equilibrium optimization method is effective in modeling practical portfolio selection problem under hybrid uncertain environment, where randomness and fuzziness are the state of affairs.

\section{Conclusions}

On the basis of probability and credibility measures, a new risk index called the ERV of random fuzzy variable was introduced. Under the equilibrium risk criterion, a new EVERV portfolio optimization model was built for portfolio selection problems, where the return rates are characterized by both probability distributions and possibility distributions. In the case where both subjective consciousness and 
TABLE 5: The optimal solutions of random model under various values of parameters.

\begin{tabular}{|c|c|c|c|c|c|c|c|}
\hline \multicolumn{2}{|c|}{ Parameters } & \multicolumn{5}{|c|}{ Investment ratios } & \multirow[t]{2}{*}{ Objective values } \\
\hline \multirow{4}{*}{$\alpha$} & \multirow{4}{*}{$\kappa$} & $x_{1}$ & $x_{2}$ & $x_{3}$ & $x_{4}$ & $x_{5}$ & \\
\hline & & $x_{6}$ & $x_{7}$ & $x_{8}$ & $x_{9}$ & $x_{10}$ & \multirow{3}{*}{$\mathrm{EV}$} \\
\hline & & $x_{11}$ & $x_{12}$ & $x_{13}$ & $x_{14}$ & $x_{15}$ & \\
\hline & & $x_{16}$ & $x_{17}$ & $x_{18}$ & $x_{19}$ & $x_{20}$ & \\
\hline \multirow{4}{*}{0.78} & \multirow{4}{*}{0.006} & 0 & 0 & 0.09906 & 0.03658 & 0 & \multirow{4}{*}{0.03408} \\
\hline & & 0.46099 & 0 & 0 & 0 & 0 & \\
\hline & & 0 & 0 & 0.06700 & 0.06212 & 0 & \\
\hline & & 0.27424 & 0 & 0 & 0 & 0 & \\
\hline \multirow{4}{*}{0.8} & \multirow{4}{*}{0.006} & 0 & 0 & 0.11689 & 0.04596 & 0 & \multirow{4}{*}{0.03398} \\
\hline & & 0.41539 & 0 & 0 & 0.01806 & 0 & \\
\hline & & 0 & 0 & 0.08538 & 0.07634 & 0 & \\
\hline & & 0.24198 & 0 & 0 & 0 & 0 & \\
\hline \multirow{4}{*}{0.82} & \multirow{4}{*}{0.006} & 0 & 0 & 0.12286 & 0.04955 & 0 & \multirow{4}{*}{0.03386} \\
\hline & & 0.37902 & 0 & 0 & 0.04157 & 0 & \\
\hline & & 0 & 0 & 0.08867 & 0.08131 & 0 & \\
\hline & & 0.21614 & 0.01161 & 0 & 0.00927 & 0 & \\
\hline \multirow{4}{*}{0.78} & \multirow{4}{*}{0.008} & 0 & 0 & 0.11519 & 0.04488 & 0 & \multirow{4}{*}{0.03399} \\
\hline & & 0.42154 & 0 & 0 & 0.01284 & 0 & \\
\hline & & 0 & 0 & 0.08459 & 0.07455 & 0 & \\
\hline & & 0.24641 & 0 & 0 & 0 & 0 & \\
\hline \multirow{4}{*}{0.78} & \multirow{4}{*}{0.009} & 0 & 0 & 0.12044 & 0.04822 & 0 & \multirow{4}{*}{0.03394} \\
\hline & & 0.40256 & 0 & 0 & 0.02893 & 0 & \\
\hline & & 0 & 0 & 0.08701 & 0.08010 & 0 & \\
\hline & & 0.23274 & 0 & 0 & 0 & 0 & \\
\hline
\end{tabular}

objective factors affect the current financial markets, the developed equilibrium framework provided a novel optimization method for depicting real-life portfolio selection problem.

When the randomness of uncertain return rates follows normal distributions, the proposed equilibrium portfolio selection model was turned into an equivalent credibilistic portfolio optimization model. The convexity of the credibilistic portfolio optimization model was discussed in Theorem 7 , which facilitates finding the desired global optimal portfolio. Furthermore, when the fuzziness of uncertain return rates follows trapezoidal, triangular, and normal distributions, the credibilistic portfolio optimization model was turned into its equivalent deterministic convex programming models.

We compared the proposed equilibrium optimization method with traditional stochastic optimization method via a portfolio selection problem. The computational results demonstrated that both optimization methods can provide diversified investment schemes. However, the obtained equilibrium optimal solutions are more superior in terms of diversification. That is, when the fuzziness of uncertain return rates is considered, the equilibrium optimal solution usually diversified the optimal solutions obtained by stochastic method. As a consequence, when the exact probability distributions of return rates are unavailable, the proposed equilibrium optimization method provided an effective way to model practical portfolio selection problems with hybrid uncertain return rates.

\section{Competing Interests}

The authors declare that they have no competing interests.

\section{Acknowledgments}

This work was supported by National Natural Science Foundation of China (no. 61374184) and the Natural Science Foundation of Hebei Province (no. A2014201166).

\section{References}

[1] H. Markowitz, "Portfolio selection," Journal of Finance, vol. 7, no. 1, pp. 77-91, 1952.

[2] H. M. Markowitz, Portfolio Selection: Efficient Diversification of Investments, John Wiley \& Sons, 1959.

[3] H. Konno and H. Yamazaki, "Mean-absolute deviation portfolio optimization model and its applications to Tokyo stock market," Management Science, vol. 37, no. 5, pp. 519-531, 1991.

[4] Y. Simaan, "Estimation risk in portfolio selection: the mean variance model versus the mean absolute deviation model," Management Science, vol. 43, no. 10, pp. 1437-1446, 1997.

[5] P. Jorion, Value at Risk: The New Benchmark for Controlling Market Risk, Irwin Professional Publishing, Willowbrook, Ill, USA, 1997.

[6] S. Y. Wang and Y. S. Xia, Portfolio Selection and Asset Pricing, Springer, Berlin, Germany, 2002. 
[7] L. Yu, S. Wang, and K. K. Lai, "Neural network-based meanvariance-skewness model for portfolio selection," Computers and Operations Research, vol. 35, no. 1, pp. 34-46, 2008.

[8] R. T. Rockafellar and S. Uryasev, "Conditional value-at-risk for general loss distributions," Journal of Banking and Finance, vol. 26, no. 7, pp. 1443-1471, 2002.

[9] A. Ang, D. Papanikolaou, and M. M. Westerfield, "Portfolio choice with illiquid assets," Management Science, vol. 60, no. 11, pp. 2737-2761, 2014.

[10] Y. Shen, X. Zhang, and T. K. Siu, "Mean-variance portfolio selection under a constant elasticity of variance model," Operations Research Letters, vol. 42, no. 5, pp. 337-342, 2014.

[11] L. A. Zadeh, "Fuzzy sets," Information and Computation, vol. 8, pp. 338-353, 1965.

[12] L. A. Zadeh, "Fuzzy sets as a basis for a theory of possibility," Fuzzy Sets and Systems, vol. 1, no. 1, pp. 3-28, 1978.

[13] J. Watada, "Fuzzy portfolio selection and its application to decision making," Tatra Mountains Mathematical Publication, vol. 13, pp. 219-248, 1997.

[14] H. Tanaka and P. Guo, "Portfolio selection based on upper and lower exponential possibility distributions," European Journal of Operational Research, vol. 114, no. 1, pp. 115-126, 1999.

[15] M. Inuiguchi and J. Ramík, "Possibilistic linear programming: a brief review of fuzzy mathematical programming and a comparison with stochastic programming in portfolio selection problem," Fuzzy Sets and Systems, vol. 111, no. 1, pp. 3-28, 2000.

[16] C. Carlsson, R. Fullér, and P. Majlender, "A possibilistic approach to selecting portfolios with highest utility score," Fuzzy Sets and Systems, vol. 131, no. 1, pp. 13-21, 2002.

[17] Y. Fang, K. K. Lai, and S.-Y. Wang, "Portfolio rebalancing model with transaction costs based on fuzzy decision theory," European Journal of Operational Research, vol. 175, no. 2, pp. 879-893, 2006.

[18] Y. Chen, Y. Liu, and J. Chen, "Fuzzy portfolio selection problems based on credibility theory," in Advances in Machine Learning and Cybernetics, vol. 3930 of Lecture Notes in Computer Science, pp. 377-386, Springer, 2006.

[19] X. Zhang, W.-G. Zhang, and R. Cai, "Portfolio adjusting optimization under credibility measures," Journal of Computational and Applied Mathematics, vol. 234, no. 5, pp. 1458-1465, 2010.

[20] Z. Qin, X. Li, and X. Ji, "Portfolio selection based on fuzzy crossentropy," Journal of Computational and Applied Mathematics, vol. 228, no. 1, pp. 139-149, 2009.

[21] H. Dastkhan, N. S. Gharneh, and H. Golmakani, "A linguisticbased portfolio selection model using weighted max-min operator and hybrid genetic algorithm," Expert Systems with Applications, vol. 38, no. 9, pp. 11735-11743, 2011.

[22] X.-L. Wu and Y.-K. Liu, "Optimizing fuzzy portfolio selection problems by parametric quadratic programming," Fuzzy Optimization and Decision Making, vol. 11, no. 4, pp. 411-449, 2012.

[23] Y. Chen, Y. Liu, and X. Wu, "A new risk criterion in fuzzy environment and its application," Applied Mathematical Modelling, vol. 36, no. 7, pp. 3007-3028, 2012.

[24] J. S. Kamdem, C. T. Deffo, and L. A. Fono, "Moments and semimoments for fuzzy portfolio selection," Insurance: Mathematics \& Economics, vol. 51, no. 3, pp. 517-530, 2012.

[25] M. K. Mehlawat and P. Gupta, "Fuzzy chance-constrained multiobjective portfolio selection model," IEEE Transactions on Fuzzy Systems, vol. 22, no. 3, pp. 653-671, 2014.

[26] X. Deng and R. Li, "Gradually tolerant constraint method for fuzzy portfolio based on possibility theory," Information Sciences, vol. 259, pp. 16-24, 2014.
[27] T. Li, W. Zhang, and W. Xu, "A fuzzy portfolio selection model with background risk," Applied Mathematics and Computation, vol. 256, pp. 505-513, 2015.

[28] E. Vercher and J. D. Bermúdez, "Portfolio optimization using a credibility mean-absolute semi-deviation model," Expert Systems with Applications, vol. 42, no. 20, pp. 7121-7131, 2015.

[29] Y. Chen and Y. Wang, "Two-stage fuzzy portfolio selection problem with transaction costs," Mathematical Problems in Engineering, vol. 2015, Article ID 675157, 12 pages, 2015.

[30] X. Huang, "Two new models for portfolio selection with stochastic returns taking fuzzy information," European Journal of Operational Research, vol. 180, no. 1, pp. 396-405, 2007.

[31] Y.-K. Liu and B. Liu, "Random fuzzy programming with chance measures defined by fuzzy integrals," Mathematical and Computer Modelling, vol. 36, no. 4-5, pp. 509-524, 2002.

[32] B. Liu, Theory and Practice of Uncertain Programming, Physica, Heidelberg, Germany, 2002.

[33] Y.-K. Liu and B. Liu, "Expected value operator of random fuzzy variable and random fuzzy expected value models," International Journal of Uncertainty, Fuzziness and Knowledge-Based Systems, vol. 11, no. 2, pp. 195-215, 2003.

[34] B. Liu and Y.-K. Liu, "Expected value of fuzzy variable and fuzzy expected value models," IEEE Transactions on Fuzzy Systems, vol. 10, no. 4, pp. 445-450, 2002.

[35] Y.-K. Liu and J. Gao, “The independence of fuzzy variables with applications to fuzzy random optimization," International Journal of Uncertainty, Fuzziness and Knowledge-Based Systems, vol. 15, pp. 1-20, 2007.

[36] Y. Liu and Y. Wang, "Equilibrium mean value of random fuzzy variable and its convergence properties," Journal of Uncertain Systems, vol. 7, no. 4, pp. 243-253, 2013. 


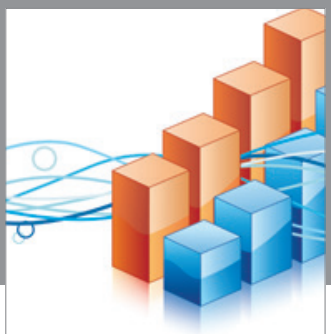

Advances in

Operations Research

vatem alat4

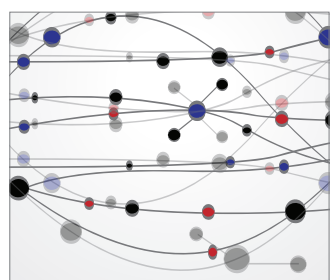

\section{The Scientific} World Journal
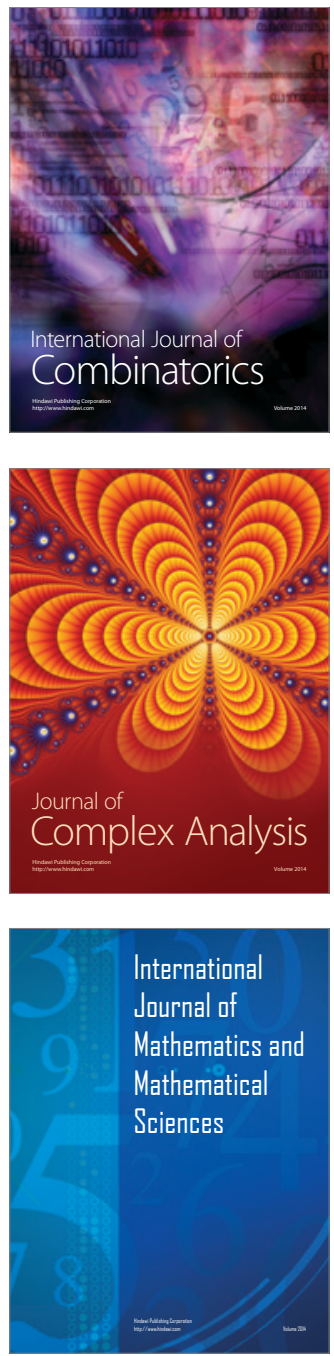
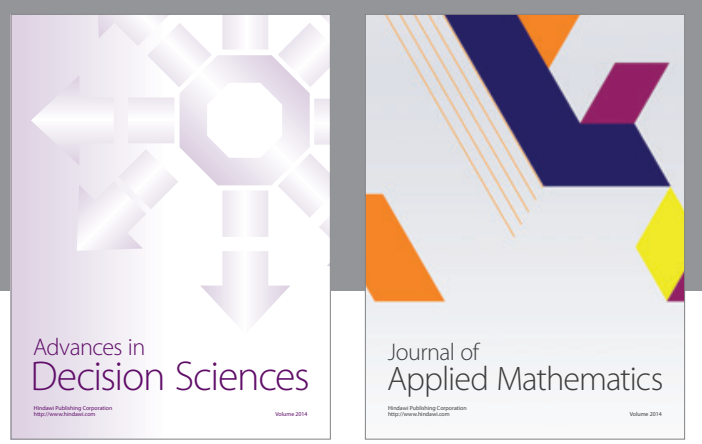

Algebra

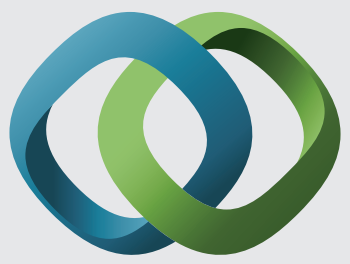

\section{Hindawi}

Submit your manuscripts at

http://www.hindawi.com
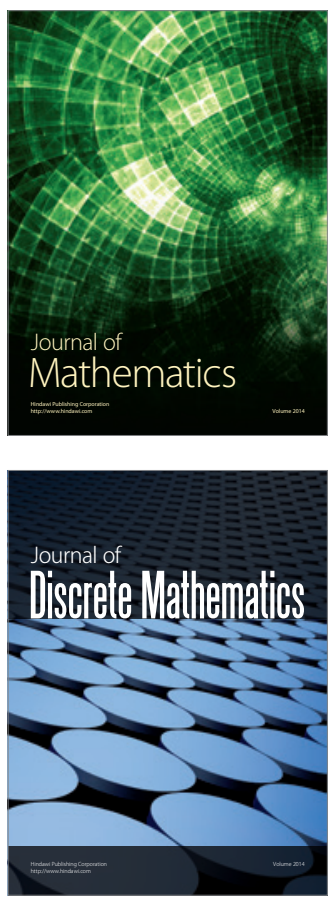

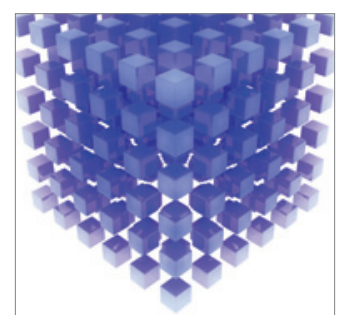

Mathematical Problems in Engineering
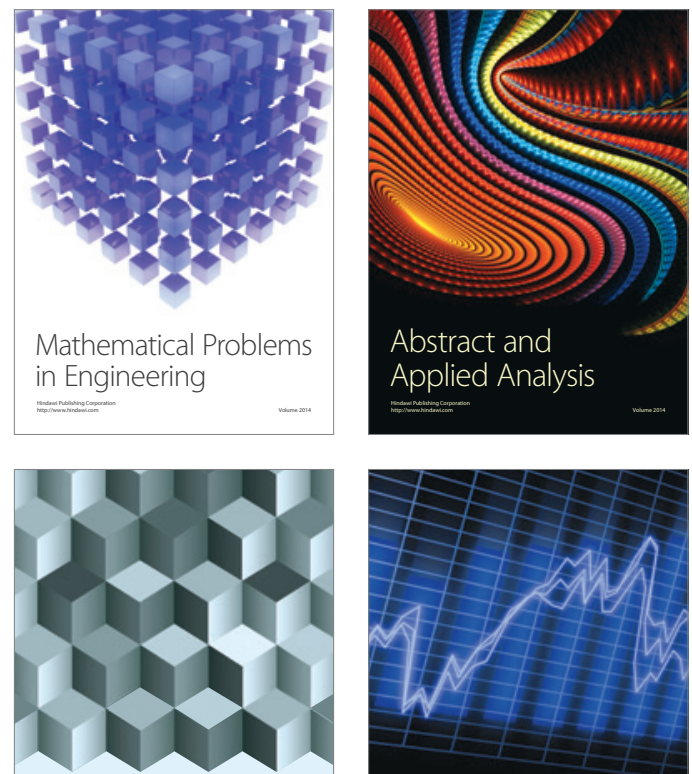

Journal of

Function Spaces

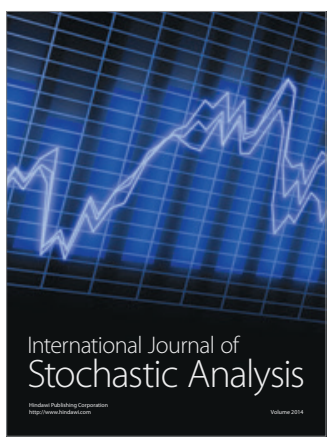

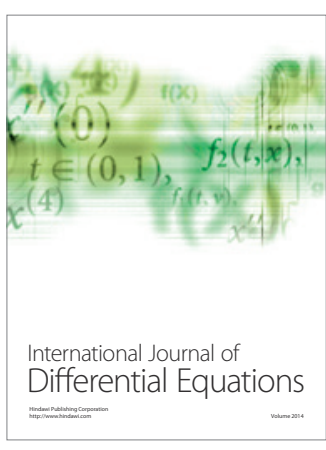
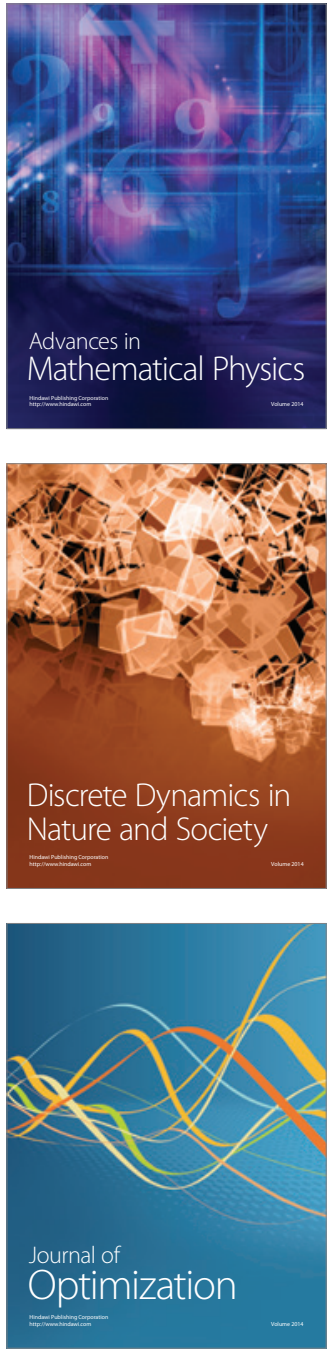